\title{
Identification of IncRNA-mRNA Regulatory Network Associated With Isolated Systolic Hypertension And Atherosclerotic Cerebral Infarction
}

Jie Yang

Shandong University of Traditional Chinese Medicine Affiliated Hospital

Yan-Nan Tao

Affiliated Second Hospital of Shandong University of traditional Chinese Medicine

Fang-Xiao Hu

Shandong University of Traditional Chinese Medicine

Yong-Zhi Chen

Shandong University of Traditional Chinese Medicine Affiliated Hospital

Xue-Song Yang

Shandong University of Traditional Chinese Medicine Affiliated Hospital

Xin Zhuang

Shandong University of Traditional Chinese Medicine Affiliated Hospital

Ming-Ling Cui

Shandong University of Traditional Chinese Medicine

Guan-lan Li

Shandong University of Traditional Chinese Medicine

Chao Li

Shandong University of Traditional Chinese Medicine

Chuan-Hua Yang ( $\square$ yangjiefenghao@163.com )

Shandong University of Traditional Chinese Medicine Affiliated Hospital https://orcid.org/0000-00033943-9451

\section{Yue-Hua Jiang}

Shandong University of Traditional Chinese Medicine Affiliated Hospital

\section{Research}

Keywords: Isolated systolic hypertension, atherosclerotic cerebral infarction, IncRNA, mRNA

Posted Date: May 25th, 2021

DOl: https://doi.org/10.21203/rs.3.rs-391143/v2 
License: (c) (i) This work is licensed under a Creative Commons Attribution 4.0 International License. Read Full License 


\section{Abstract}

Background: Increasing evidences uncover that IncRNAs play an important role in Isolated systolic hypertension (ISH). However, a systematic IncRNA-mRNA regulatory network is still absent in isolated systolic hypertension and atherosclerotic cerebral infarction patients (ISH \& ACl).

Aim:This research aims to establish a IncRNA-mRNA co-expression network in patients with ISH \& ACl, to probe into the potential functions of IncRNA in those patients.

Design and Setting:Expression profiles of IncRNA and mRNAs are collected and compared respectively from 8 patients with ISH and 8 patients with ISH \& ACI by RNA-seq data.

Methods: Differentially expressed IncRNAs and mRNAs were screened out via high-throughput sequencing in the plasma of ISH/ACl patients and control ISH patients. Then, a IncRNA-mRNA interaction network was built using the Pearson correlation coefficient by Cytoscape software. The expression levels of the hub genes and IncRNAs were verified by qRT-PCR in another $10 \mathrm{ISH} / \mathrm{ACl}$ patients and 10 control patients.

Results: 2768 differentially expressed IncRNAs and 747 differentially expressed mRNAs were identified. 2 hub genes (CD226 and PARVB) and 11 IncRNAs were identified in the IncRNA-mRNA interaction network. qRT-PCR and cell assay results verified that IncRNAs ENST00000590604 and CD226 are highly expressed in patients of ISH \& ACl. CD226 was associated with vascular endothelial cells growth and stability through platelet activation and focal adhesion pathway.

Conclusion: We established a novel mRNA-IncRNA interaction network. IncRNAs ENST00000590604 and CD226 might be the potential biomarkers of ISH \& ACI.

\section{How This Fits In}

Occurrences of cerebral infarction are closely related to hypertension and wide pulse pressures have become an independent predictor for cardiovascular morbidity. However, a systematic IncRNA-mRNA regulatory network is still absent in isolated systolic hypertension and atherosclerotic cerebral infarction patients.

This paper indicates that IncRNAs ENST00000590604 and CD226 might be the potential biomarkers of ISH\&ACI patients. In a clinical context, these IncRNAs can be adopted as biomarkers for precaution of ACI in ISH patients.

\section{Introduction}

Isolated systolic hypertension (ISH) is a major risk factor for cardiovascular morbidity and mortality, and the most common form of hypertension in the elderly population [1, 2]. With an ageing population, the prevalence of hypertension, particularly ISH, is rapidly rising [2]. Besides, ISH is associated with 
cerebrovascular diseases, such as Isolated Systolic Hypertension and Atherosclerotic Cerebral Infarction patients (ISH\&ACI) [2].

However, the underlying molecular mechanisms of ISH\&ACl have not been elucidated. Therefore, we seek the key molecules involved in ISH\&ACl and identify potential diagnostic biomarkers and therapeutic targets for ISH\&ACl.

Long non-coding RNAs (IncRNAs) is a class of IncRNA longer than 200 nucleotides. LncRNAs are involved in many cellular processes, including chromatin remodeling, transcription modulation, and interacting with other RNAs to modulate gene expression [3]. With the rapid technological advances in high-throughput sequencing, the research of IncRNAs has made remarkable progress [4]. A lot of experimental evidence shows that IncRNAs play an important role in the development of hypertension and might be the potential key regulators of hypertension [5, 6]. LncRNAs TUG1/miR-145-5p/FGF10 regulates the proliferation and migration of vascular smooth muscle cell in hypertension by activating the Wnt/ $\beta$-catenin pathway [7]. Down-regulation of MRAK048635_P1 is probably an important factor for vascular remodeling in essential hypertension [8]. Nevertheless, the relationship between IncRNAs and ISH has not been reported.

In this paper, 946 up-regulated and 1822 down-regulated differentially expressed IncRNAs, and 288 upregulated and 519 down-regulated differentially expressed mRNAs were identified between ISH\&ACI cases and ISH controls, respectively. The GO and KEGG enrichment analyses revealed that differentially expressed IncRNAs and mRNAs were significantly enriched in platelet activation. According to the IncRNA-mRNA co-expression network, 2 hub genes (CD226 and PARVB) were identified and 9 IncRNAs were predicted to target these 2 hub genes. Then, the IncRNA-mRNA pairs were verified by real-time PCR in 20 individual samples. Besides, flow cytometer was performed to evaluate proliferation and apoptosis rate and real-time PCR was performed to validate the IncRNA-mRNA pairs in Human Brain Microvascular Endothelial Cells (HBMECs).

In summary, our results showed that IncRNAs ENST00000590604 regulated the expression of CD226 through platelet activation and focal adhesion pathway. IncRNAs ENST00000590604 and CD226 might be the potential biomarkers of isolated systolic hypertension and atherosclerotic cerebral infarction patients.

\section{Materials And Methods}

\subsection{Study samples}

A total of 18 ISH\&ACl patients were recruited from the Affiliated Hospital of Shandong University of Traditional Chinese Medicine from May 2019 to April 2020. Isolated systolic hypertension and atherosclerotic cerebral infarction were defined according to the 2018 revised edition of the Chinese guidelines for the prevention and treatment of hypertension (exposure draft) and 2015 China Cerebrovascular Disease Classification. Meanwhile, subjects conforming to the following standards were 
included: 1. aged 18 years old or above, no gender limitations; 2. within 2 weeks to 6 months after the occurrence of cerebral infarction; 3 . willing to participate in this experiment and cooperate with the doctors and sign the informed consent in writing. A total of 18 isolated systolic hypertension (ISH) controls were recruited from the same hospital during the same period. 8 ISH\&ACl patients and $8 \mathrm{ISH}$ controls were randomly selected from 18 ISH\&ACl patients and 18 ISH controls for RNA sequencing, and the remaining samples were used as replication population for the validation of differentially expressed IncRNAs and mRNAs.

The study protocol was approved by the Ethics Committee of the Affiliated Hospital of Shandong University of Traditional Chinese Medicine in Shandong, China. Written informed consent was obtained from all patients or their families under the Declaration of Helsinki.

\subsection{RNA extraction, library preparation, and RNA-seq}

RNA was extracted from plasma using the QIAamp Circulating Nucleic Acid Kit (QIAGEN, Germany) according to the manufacturer's protocol. RNA was then purified and concentrated using the RNeasy MinElute Cleanup Kit (QIAGEN, Germany). RNA quality was evaluated using NanoDrop 2000 (Thermo Fisher, USA). Sequencing libraries were generated using TruSeq Stranded Total RNA LT - (with Ribo - Zero TM Gold) - Set A (Illumina, USA) following the manufacturer's recommendations. The libraries were then sequenced on the Illumina Hiseq 2500 platform and 125bp/150bp paired-end reads were generated.

\subsection{Quantitative real-time PCR (qRT-PCR) validation}

qRT-PCR was used to validate selected IncRNAs and mRNAs in 20 individual samples. Reverse transcription was performed using the PrimeScript ${ }^{\text {TM }}$ RT reagent Kit with gDNA Eraser (Sparkjade, Shandong, China). qRT-PCR was performed using a TB Green ${ }^{\circledR}$ Premix Ex Taq ${ }^{\text {TM }}$ II (Sparkjade, Shandong, China) and an Applied Biosystems 7500 Real-Time PCR System (Thermo Fisher, USA) according to standard methods. Specific primers of IncRNAs and mRNAs were listed in Supplementary Table 1. The 2$\triangle \triangle C T$ method was used to calculate the relative fold-change to the house-keeping gene ( $\beta$-action).

\subsection{Cell}

Cell culture: HBMECs (Hunan Fenghui Biotechnology Co.Ltd,Cat.CL0116) were cultured with high glucose's (4.5 mg/ml) Dulbecco's Modified Eagle Medium (DMEM, Gibco, REF.\#C11995065BT) and supplemented with 10\% Fetal Bovine Serum (FBS, Gibco, REF.10099141) and 1\% Penicillin/Streptomycin solution (P/S, HyClone, Cat. \#SV30010) at $37^{\circ} \mathrm{C}$ in a humidified $5 \% \mathrm{CO}_{2}$ incubator. Then, HBMECs were intervened by Sodium pyruvate solution Ang II (Solarbio Co., Ltd., Beijing, China) and OGD/R (Oxygen-

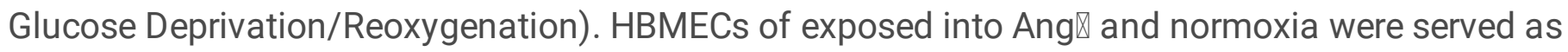
controls.

MTT assay: The effect of Ang $\varangle$ and OGD/R on proliferation were detected by MTT assay. HBMECs were seeded at a density of 1500 cells per well into 96-well microplates in a final volume of $100 \mu \mathrm{L}$ with $2 \times 10^{-}$

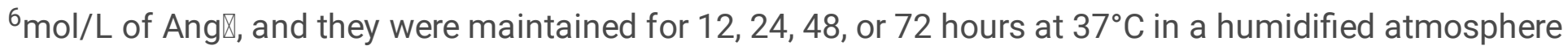


containing $5 \% \mathrm{CO}_{2}$. After that, $\mathrm{HBMECs}$ were cultured in Earle's Balanced Salt Solution (EBSS, Solarbio, Cat.H2025) without glucose and fetal bovine serum and deprived-oxygen by transferring into an anaerobic incubator $\left(0 \% \mathrm{O}_{2} / 5 \% \mathrm{CO}_{2} / 95 \% \mathrm{~N}_{2}\right)$ for 4 hours. After OGD, cells were returned to a normoxic incubator under $5 \% \mathrm{CO}_{2} / 95 \%$ air for 24 hours in complete medium. Subsequently, to monitor cell viability, $10 \mu$ of sterile 3-(4,5-dimethylthiazol-2-yl)-2,5-Diphenyl-2H-tetrazolium bromide dye (MTT; Solarbio, Lot.\#303H0525, Beijing, China) at a final concentration of $5 \mathrm{mg} / \mathrm{ml}$ was added to each well for incubation at $37^{\circ} \mathrm{C}$ for $4 \mathrm{~h}$; this was followed by the administration of $150 \mu \mathrm{l}$ dimethyl sulfoxide solution (DMSO; Sinopharm Chemical Reagent Co., Ltd, Cat. \#30072418, Shanghai, China) per well to dissolve the formazan crystals. The optical density value (OD-value) was measured at $562 \mathrm{~nm}$ using a Multiskan GO microplate reader (Thermo Scientifific, Waltham, MA, USA) according to the manufacturer's instruction. The OD-value indicates the counts of living cells.

Apoptosis rate assay: The effect of Ang $\nabla$ and $O G D / R$ on apoptosis rate was assessed using Annxin $V$ staining by Muse Cell Analyer(Germany Merck\&Millipore), according to the manufacturer's direction.

qRT-PCR: To validate the sequence data, $11 \mathrm{DE}$ genes related to endothelial dysfunction were chosen for qRT-PCR, including 8 IncRNAs (ENST00000565493,ENST00000606054,ENST00000590604,NONHSAT138949.2, ENST00000566942, ENST00000369385, NONHSAT217189.1, ENST00000540082) and their corresponding target mRNAs (CD226,PARVB). After being cultured in 6-well plates at a density of $6000 \mathrm{cells} / \mathrm{cm}^{2}$, total RNAs from model cells and control cells were extracted using SPARKeasy Improved Tissue/Cell RNA Kit (HAOSAIL, Lot.YADKZ). PrimeScript ${ }^{\text {TM }}$ RT reagent Kit with gDNA Eraser (Perfect Real Time) (TaKaRa, Cat.\#RR047A) and reverse transcription reaction according to thestandard operation process provided by the manufacturer was used to synthesize the DNA template. TB Green ${ }^{T M}$ Premix Ex Taq ${ }^{\mathrm{TM}}$ II (Tli RNaseH Plus) (TaKaRa, Cat.\#RR820A) was used to amplify qRT-PCR following the manufacturer'sinstructions. LncRNAs expression was normalized to GAPDH as described inthe literature. Complementary DNA was synthesized using a Transcriptor First Strand cDNA Synthesis Kit (Roche, Cat. \#04379012001, Germany), and qRTPCR was performed by using LightCycler 480 SYBR Green I Master (Roche, Cat. \#04707516001), according to the manufacturer's instructions, using a LightCycler480 instrument (Roche). The primer sequences are listed in SupplementaryTable 1 (Sangon Biotech, Shanghai, China). The data were calculated and presented by the $2^{-\triangle \Delta C T}$ method.

\subsection{Statistical analysis}

All statistical analyses were performed using the R 3.6.0. Two-tailed student t-test was used for normally distributed variables and the Wilcoxon Rank-Sum test was used for abnormally distributed data. Data normality was evaluated using the Shapiro-Wilk test. Differences between case and control group were considered significant at $\mathrm{p}$-value $₫ 0.05$.

\section{Results}




\subsection{Identification of DEGs and DELs}

Read counts obtained from RNA-seq were normalized as fragments per kilobase of transcript per million mapped fragments (FPKM) [9]. FPKM was used to identify the differentially expressed IncRNAs and mRNAs in ISH\&ACl cases $(n=8)$ and ISH controls $(n=8)$. The differentially expressed IncRNAs and mRNAs meted the following cutoff criteria: p-value $\mathbb{0} 0.05$ and |Fold-Change|>2 [10]. A total of 2768 differentially expressed IncRNAs (946 up-regulated and 1822 down-regulated) and 747 differentially expressed mRNAs (288 up-regulated and 519 down-regulated) were identified between ISH\&ACl cases and ISH controls. Volcano plots visualized the distribution of the differentially expressed IncRNAs (Fig. 1A) and mRNAs (Fig. 1B). The hierarchical clustering plots showed clear separation and consistency in the expression profiles of ISH\&ACl cases and ISH controls (Fig. 1C and Fig. 1D).(Fig. 1 Differentially expressed IncRNAs and mRNAs in ISH\&ACl cases $(n=8)$ and ISH controls $(n=8)$. (A)Volcano plots of differentially expressed IncRNAs. The X-axis is log2 Fold-Change and the Y-axis is log10p-value. Red (upregulated) and blue (down-regulated) points indicated the differential expression IncRNA. (B) Volcano plots of differentially expressed mRNAs.(C)Differentially expressed IncRNAs hierarchically clustered, each column represents a sample and every row represents a IncRNA. Red indicates high relative expression and blue indicates low relative expression. (D) Differentially expressed mRNAs hierarchically clustered.)

\subsection{Gene Ontology (GO) and KEGG pathway analysis}

GO enrichment analysis and KEGG enrichment analysis were performed by hypergeometric test to find significantly enriched GO terms and KEGG pathways of differentially expressed IncRNAs and mRNAs. By GO enrichment analysis, differentially expressed IncRNAs and mRNAs were classified into three categories: cellular component (CC), molecular function (MF), and biological process (BP). The potential mRNAs targets of differentially expressed IncRNAs were predicted using cis-specific binding modules and trans-specific binding modules. The intersection of targets of differentially expressed IncRNAs and differentially expressed mRNAs were used to perform enrichment analysis.

As shown in Fig. 2A and Supplementary Table S2, GO Enrichment analysis identified 42 significant GO terms ( $p$-value $\leq 0.05$ ). In biological process, the top four $\mathrm{GO}$ terms were positive regulation of blood coagulation, positive regulation of coagulation, cellular response to glucagon stimulus, and platelet degranulation; In cellular component, the top four GO terms were platelet alpha granule, platelet alpha granule lumen, secretory granule lumen, and cytoplasmic membrane-bounded vesicle lumen; In molecular function, the top two GO terms were integrin binding and cell adhesion molecule binding. KEGG enrichment analysis identified 6 significant KEGG pathways (Fig. 2B and Supplementary Table S3). The 6 significant KEGG pathways were arrhythmogenic right ventricular cardiomyopathy, platelet activation, ECM-receptor interaction, hypertrophic cardiomyopathy, dilated cardiomyopathy, and Focal adhesion. The enrichment analyses showed that the intersection of targets of differentially expressed IncRNAs and differentially expressed mRNAs were mainly associated with platelet activation.(Fig. 2 GO and KEGG pathway enrichment analysis.GO enrichment contains three terms: cellular component, molecular function, and biological process. (B) the top 30 enriched KEGG pathways.). 


\subsection{IncRNA-mRNA co-expression network construction and hub genes analysis}

We calculated the Pearson correlation coefficient using the expression value of IncRNAs and mRNAs. We identified IncRNA-mRNA co-expression with coefficient great than 0.9 and the p-value less than 0.05 . According to the results of IncRNA-mRNA co-expression, we then constructed the IncRNA-mRNA network using Cytoscape software. 2 hub genes in the network were identified and the 2 hub genes were CD226 and PARVB (Fig. 3). (Fig. 3. IncRNA-mRNA co-expression network. Red indicates IncRNAs and blue indicates mRNAs. the top 2 hub genes are CD226 and PARVB.)

CD226 (also known as DNAM-1, PTA-1, or TLiSA-1) was an immunoglobulin-like glycoprotein. CD226 was expressed on the platelet membrane and was involved in platelet activation [11, 12]. Platelets played a crucial role in the control of acute hemorrhage and thrombosis, and thus were involved in atherosclerosis and myocardial infarction [13]. CD226 may participate in atherosclerotic cerebral infarction. The PARVB (also known as beta-parvin and affixin) encoded a member of the parvin family of actin-binding proteins, which played a role in cytoskeleton organization and cell adhesion [14]. PARVB was shown to be associated with coronary restenosis, hematoma volume, and spontaneous intracerebral hemorrhage $[15,16]$.

\subsection{The validation of expression profiles of IncRNAs}

qRT-PCR was performed for further validation of 9 selected differentially expressed IncRNA-mRNA pairs in 20 individual samples (Table 1). We found that the expression of CD226 and IncRNAs

ENST00000590604 were significantly up-regulated in the cases compared to controls (Fig. 4). The results of IncRNAs ENST00000590604 and CD226 were consistent with the results of RNA-seq data. Hub gene CD226 was significantly upregulated in ISH\&ACl patients.(Fig. 4. Relative levels of mRNAs and IncRNAs in 10 cases and 10 controls. Using qRT-PCR to calculate the relative fold-change to the house-keeping gene ( $\beta$-action). From left to right are IncRNA ENST00000590604 and CD226.)

Table 1

-differentially expressed IncRNA-mRNA pairs

\begin{tabular}{|ll|}
\hline Target & IncRNAs \\
\hline CD226 & $\begin{array}{l}\text { ENST00000565493, ENST00000606054, ENST00000590604, NONHSAT217189.1, } \\
\text { ENST00000566942 }\end{array}$
\end{tabular}

PARVB ENST00000565493, NONHSAT138949.2, ENST00000369385, ENST00000540082

\subsection{The effects of Ang囚 and OGD/R on HBMECs}

MTT assay and flow cytometer (FCM) were performed to evaluate proliferation and apoptosis rate. The results indicated that, compared with the control group, HBMECs' growth was inhibited and apoptosis rate was increased which were treated with OGD/R (Fig. 5).(Fig. 5. The effect of OGD/R on proliferation and apoptosis rate of HBMECs. (A)HBMECs' viability was declined after OGD/R compared with control 
group ( ${ }^{*} \mathrm{P}<0.05$, ${ }^{\star *} \mathrm{P}<0.01$ vs. Control group). (B) The apoptosis rate of OGD/R group was increased compared with control group ( ${ }^{\star *} \mathrm{P}<0.01$ vs. Control group). (C) Control group apoptosis results. (D) OGD/R group apoptosis results.)

\section{5 qRT-PCR validation of significant DE IncRNAs and DE mRNAs of HBMECs}

The qRT-PCR results using HBMECs were consistent with the RNA-Seq results and qRT-PCR results using plasma of patients, including IncRNA ENST00000590604 and CD226 (Fig. 6).(Fig. 6. qRT-PCR validation $(n=3)$ of DE genes compared to RNA-Seq $(n=8)$. Yellow represents the expression of DE IncRNAs and DE mRNAs in the ISH complicated with stroke group veeq, blue represents the expression of DE IncRNAs and

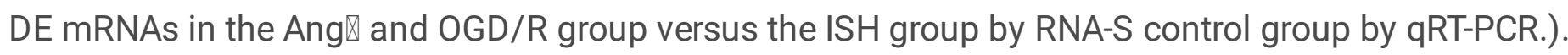

\section{Discussion}

\subsection{Summary}

Hypertension becomes one of the most important risk factors for cardiovascular and cerebrovascular accidents in the world today and contributes to an elevated risk of stroke, myocardial infarction, heart failure, and renal failure $[17,18] .31 .1 \%$ of adults had hypertension in the whole world [19]. The health cost of hypertension has an enormous public health burden. ISH is the most common type of hypertension in elderly men as well as young adults [20].

The emerging evidence indicates that IncRNAs play a critical role in various biological functions and disease processes [21]. For example, HOTAIR (HOX transcript antisense intergenic RNA) is one of the most well-studied IncRNAs that are overexpressed in a variety of cancers [22]. Though the significance of IncRNAs is being increasingly found in hypertension, the expression profile and the mechanism of IncRNAs remain unclear in ISH.

In this study, our RNA-seq data revealed multiple IncRNAs dysregulated in ISH\&ACI. GO and KEGG enrichment analyses found that the intersection of the targets of differentially expressed IncRNAs and differentially expressed mRNAs were mainly associated with platelet activation, ECM-receptor interaction, and focal adhesion. Platelet activation signaling pathway played a critical role in the function of platelets in hemostasis and thrombosis [23]. Activated platelets aggregated at the site of endothelial cell erosion, stimulating thrombus formation and promoting atherothrombotic disease [24]. Focal adhesion signaling pathway promoted cell migration and angiogenesis $[25,26]$.

We also found two upregulated hub genes in ISH\&ACI (CD226 and PARVB) by the IncRNA-mRNA coexpression network. CD226 was an immunoglobulin-like glycoprotein and mediated platelet and megakaryocytic cell adhesion to vascular endothelial cells $[12,27]$. Researches suggested that plateletderived miR-142-3p regulating apoptosis of endothelial cells in hypertension and the dysfunction of vascular endothelial cells played crucial roles in vascular remodeling during hypertension [28, 29]. PARVB 
affected human endothelial cell shape and motility, suggesting an important role of PARVB in vessel growth and vessel stability [30, 31].

We further validated the results by qRT-PCR in another group of 20 patients. Besides, flow cytometer was performed to evaluate proliferation and apoptosis rate in HBMECs cells and real-time PCR was performed to validate the IncRNA-mRNA pairs in HBMECs cells. Here, we found IncRNAs ST00000590604 regulated the expression of CD226. CD226 was associated with vascular endothelial cells growth and stability through platelet activation and focal adhesion pathway. IncRNAs ENST00000590604 and CD226 could be the potential biomarkers for ISH\&ACI and provided a new idea for the clinical treatment of ISH\&ACl.

\subsection{Limitations}

There were several limitations:

The cases included in this research are limited in number so that expansion of sampling size is necessary for future research to generate more reliable results. Also, since no medication targeted the differentially expressed IncRNAs or mRNAs, further research on the pharmacological mechanism are still needed to unveil the essential roles of these potential biomarkers.

\subsection{Comparison with existing literature}

Past research mostly focused on the differential expressions of IncRNAs and mRNAs in ACl patients and healthy subjects or in hypertension patients and healthy subjects. The differential expressions of IncRNAs in ISH\&ACI patients and ISH patients have not been investigated. We initiated the probe into the differentially expressed IncRNAs and mRNAs in ISH\&ACI patients and ISH patients at the transcriptive level in hope of demonstrating the potential mechanism for the progression of the disease.

\subsection{Implications for Research and Practice}

The findings of the experiment allow discussion of the physiological foundation for ISH\&ACl from the level of IncRNAs via high-through sequencing. We drew a preliminary conclusion on the potential pathomechanism for ISH patients to lapse into $\mathrm{ACl}$. Our research provided theoretical foundation for future precise diagnoses and treatments with medication.

\section{Conclusion}

We established a novel mRNA-IncRNA interaction network. IncRNAs ENST00000590604 and CD226 might be the potential biomarkers of ISH \& ACl. This exploratory work generates a novel mRNA-IncRNA interaction network that was never before carried out in ISH\&ACI patients. The two IncRNAs ENST00000590604 and CD226 verified as potential biomarkers for ISH\&ACI were not mentioned in former literature.

\section{Abbreviations}




\begin{tabular}{|ll|}
\hline ISH & Isolated systolic hypertension \\
\hline ISH/ACI & Isolated systolic hypertension and atherosclerotic cerebral infarction patients \\
\hline IncRNAs & Long non-coding RNAs \\
\hline HBMECs & Human Brain Microvascular Endothelial Cells \\
\hline OGD/R & Oxygen-Glucose Deprivation/Reoxygenation \\
\hline OD-value & optical density value \\
\hline FPKM & transcript per million mapped fragments \\
\hline KEGG & Kyoto Encyclopedia of Genes and Genomes \\
\hline CC & cellular component \\
\hline MF & molecular function \\
\hline BP & biological process \\
\hline GO & Gene Ontology \\
\hline FCM & flow cytometer \\
\hline HOTAIR & HOX transcript antisense intergenic RNA \\
\hline
\end{tabular}

\section{Declarations}

\section{Ethics approval and consent to participate}

We have obtained the ethic clearance certificate and all the participants signed the informed consent.

\section{Availability of data and materials}

The datasets used and/or analysed during the current study are available from the corresponding author on reasonable request.

\section{Conflicts of interest}

The author of this article has no conflict of interest

\section{Funding}

This work was supported by the National Natural Science Foundation of China (No.81804061); Shandong Province 'Taishan Scholar' Construction ProjectFunds No.2018-35; Shandong Traditional Chinese Medicine Science and Technology Development Project (No.2019-0095); Ji'nan Science and Technology Project(No.201805078) 
All participants signed the informed consent

\section{Consent for publication}

Not Applicable

\section{Authors' contributions}

YJ,TYN,HFX,CML,LGL,LC and JYH performed the experiments and drafted the manuscript. CYZ and YXS participated in the data interpretation and discussion. ZX contributed to software applications and data analysis. YJ and $\mathrm{YCH}$ designed the study and carried out important revisions of the manuscript. All authors read and approved the final manuscript.All authors read and approved the final manuscript.

\section{References}

1. Mancia G, Giannattasio C: Diagnostic and therapeutic problems of isolated systolic hypertension. J Hypertens,2015, 33:33-43.

2. Bavishi C, Goel S, Messerli FH: Isolated Systolic Hypertension: An Update After SPRINT. Am J Med,2016, 129:1251-1258.

3. Lorenzen JM, Thum T: Long noncoding RNAs in kidney and cardiovascular diseases. Nat Rev Nephrol,2016, 12:360-373.

4. Jathar S, Kumar V, Srivastava J, Tripathi V: Technological Developments in IncRNA Biology. Adv Exp Med Biol,2017, 1008:283-323.

5. Jusic A, Devaux Y: Noncoding RNAs in Hypertension. Hypertension,2019, 74:477-492.

6. Shi L, Tian C, Sun L, Cao F, Meng Z: The IncRNA TUG1/miR-145-5p/FGF10 regulates proliferation and migration in VSMCs of hypertension. Biochemical and Biophysical Research Communications,2018, 501:688-695.

7. Shi L, Tian C, Sun L, Cao F, Meng Z: The IncRNA TUG1/miR-145-5p/FGF10 regulates proliferation and migration in VSMCs of hypertension. Biochem Biophys Res Commun,2018, 501:688-695.

8. Fang G, Qi J, Huang L, Zhao X: LncRNA MRAK048635_P1 is critical for vascular smooth muscle cell function and phenotypic switching in essential hypertension. Biosci Rep 2019, 39.

9. Mortazavi A, Williams BA, McCue K, Schaeffer L, Wold B: Mapping and quantifying mammalian transcriptomes by RNA-Seq. Nat Methods,2008, 5:621-628.

10. Zhao X, Tang DY, Zuo X, Zhang TD, Wang C: Identification of IncRNA-miRNA-mRNA regulatory network associated with epithelial ovarian cancer cisplatin-resistant. J Cell Physiol ,2019, 234:1988619894.

11. Scott JL, Dunn SM, Jin B, Hillam AJ, Walton S, Berndt MC, Murray AW, Krissansen GW, Burns GF: Characterization of a novel membrane glycoprotein involved in platelet activation. J Biol Chem,1989, 264:13475-13482. 
12. Kojima H, Kanada H, Shimizu S, Kasama E, Shibuya K, Nakauchi H, Nagasawa T, Shibuya A: CD226 mediates platelet and megakaryocytic cell adhesion to vascular endothelial cells. J Biol Chem,2003, 278:36748-36753.

13. Zhang J, Ding Y, Jiang D, Xie J, Liu Y, Ma J, Mu Y, Zhang X, Yu C, Zhang Y, et al: Deficiency of platelet adhesion molecule CD226 causes megakaryocyte development and platelet hyperactivity. Faseb j,2020, 34:6871-6887.

14. Yamaji S, Suzuki A, Sugiyama Y, Koide Y, Yoshida M, Kanamori H, Mohri H, Ohno S, Ishigatsubo Y: A novel integrin-linked kinase-binding protein, affixin, is involved in the early stage of cell-substrate interaction. J Cell Biol,2001, 153:1251-1264.

15. Marini S, Devan WJ, Radmanesh F, Miyares L, Poterba T, Hansen BM, Norrving B, Jimenez-Conde J, Giralt-Steinhauer E, Elosua R, et al: 17p12 Influences Hematoma Volume and Outcome in Spontaneous Intracerebral Hemorrhage. Stroke,2018, 49:1618-1625.

16. Verschuren JJ, Trompet S, Sampietro ML, Heijmans BT, Koch W, Kastrati A, Houwing-Duistermaat JJ, Slagboom PE, Quax PH, Jukema JW: Pathway analysis using genome-wide association study data for coronary restenosis--a potential role for the PARVB gene. PLoS One, 2013, 8:e70676.

17. Lawes CM, Vander Hoorn S, Rodgers A: Global burden of blood-pressure-related disease, 2001. Lancet,2008, 371:1513-1518.

18. Li W, Gu H, Teo KK, Bo J, Wang Y, Yang J, Wang X, Zhang H, Sun Y, Jia X, et al: Hypertension prevalence, awareness, treatment, and control in 115 rural and urban communities involving 47000 people from China. J Hypertens,2016, 34:39-46.

19. Mills KT, Bundy JD, Kelly TN, Reed JE, Kearney PM, Reynolds K, Chen J, He J: Global Disparities of Hypertension Prevalence and Control: A Systematic Analysis of Population-Based Studies From 90 Countries. Circulation,2016, 134:441-450.

20. Kobalava ZD, Kotovskaya YV: [Isolated Systolic Hypertension in Different Ages]. Kardiologiia ,2015, 55:84-90.

21. Peng WX, Koirala P, Mo YY: LncRNA-mediated regulation of cell signaling in cancer. Oncogene,2017, 36:5661-5667.

22. Bhan A, Soleimani M, Mandal SS: Long Noncoding RNA and Cancer: A New Paradigm. Cancer Research,2017, 77:3965-3981.

23. Estevez B, Du X: New Concepts and Mechanisms of Platelet Activation Signaling. Physiology (Bethesda) ,2017, 32:162-177.

24. Yun SH, Sim EH, Goh RY, Park JI, Han JY: Platelet Activation: The Mechanisms and Potential Biomarkers. Biomed Res Int,2016, 2016:9060143.

25. Zhao X, Guan JL: Focal adhesion kinase and its signaling pathways in cell migration and angiogenesis. Adv Drug Deliv Rev,2011, 63:610-615.

26. Liu B, Xu L, Yu X, Jiao X, Yan J, Li W, Guo M: Genistein Inhibited Estradiol-Induced Vascular Endothelial Cell Injury by Downregulating the FAK/Focal Adhesion Pathway. Cell Physiol Biochem,2018, 49:2277-2292. 
27. Chen L, Xie X, Zhang X, Jia W, Jian J, Song C, Jin B: The expression, regulation and adhesion function of a novel CD molecule, CD226, on human endothelial cells. Life Sci,2003, 73:2373-2382.

28. Bao H, Yao QP, Huang K, Chen XH, Han Y, Jiang ZL, Gao LZ, Qi YX: Platelet-derived miR-142-3p induces apoptosis of endothelial cells in hypertension. Cell Mol Biol (Noisy-le-grand) ,2017, 63:3-9.

29. Blann AD, Nadar S, Lip GY: Pharmacological modulation of platelet function in hypertension. Hypertension,2003, 42:1-7.

30. Javerzat S, Franco M, Herbert J, Platonova N, Peille AL, Pantesco V, De Vos J, Assou S, Bicknell R, Bikfalvi A, Hagedorn M: Correlating global gene regulation to angiogenesis in the developing chick extra-embryonic vascular system. PLoS One,2009, 4:e7856.

31. Pitter B, Werner AC, Montanez E: Parvins Are Required for Endothelial Cell-Cell Junctions and Cell Polarity During Embryonic Blood Vessel Formation. Arterioscler Thromb Vasc Biol,2018, 38:11471158.

\section{Supplemental Data}

Supplemental Tables 1-3 are not available with this version.

\section{Figures}


(A)

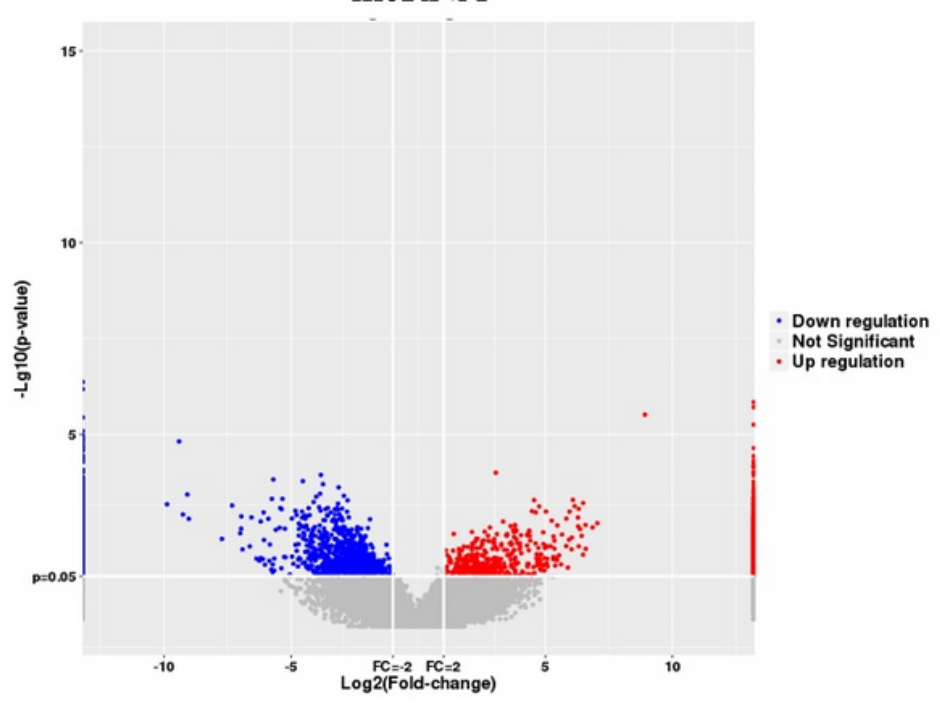

(C)

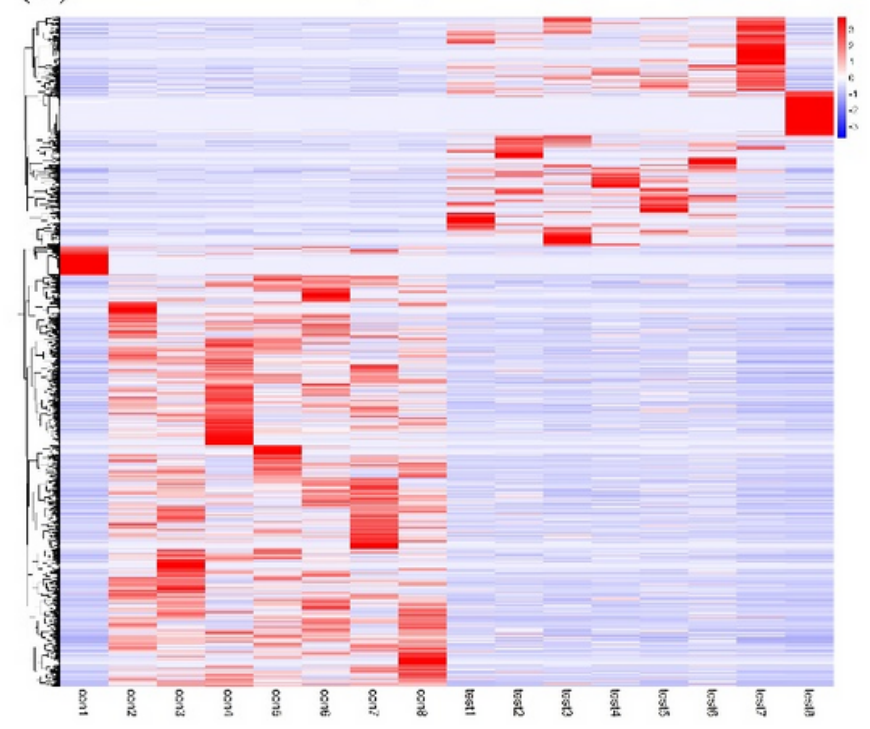

(B)

mRNAs

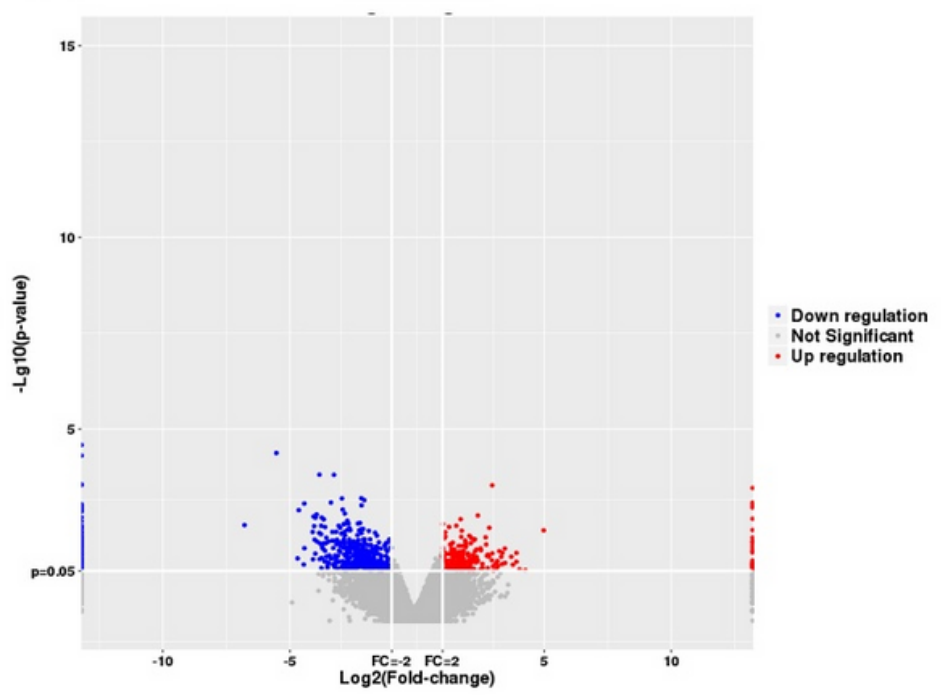

(D)
mRNAs

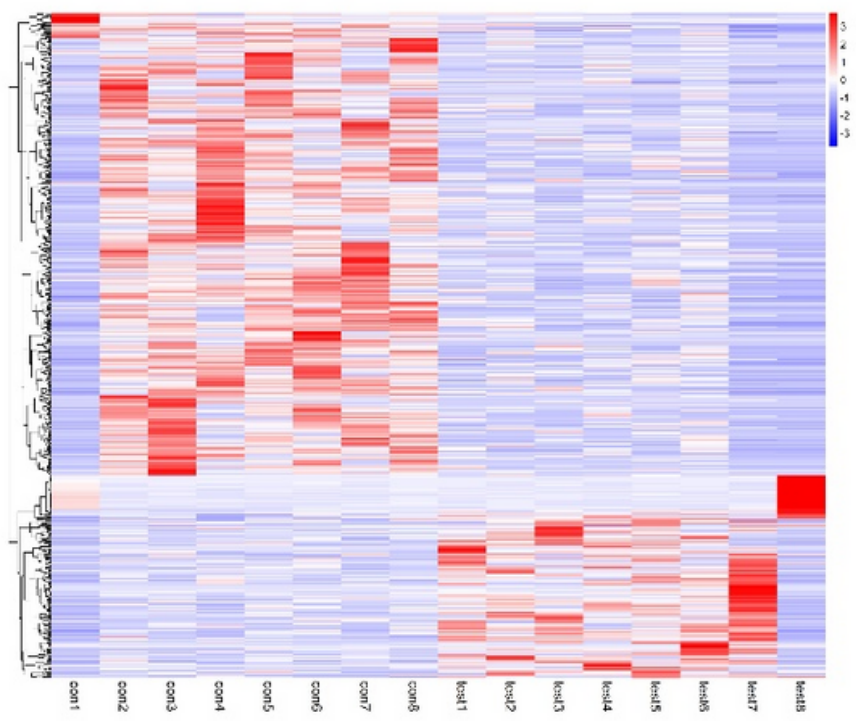

\section{Figure 1}

Differentially expressed IncRNAs and mRNAs in ISH\&ACI cases $(n=8)$ and ISH controls $(n=8)$. (A) Volcano plots of differentially expressed IncRNAs. The X-axis is log2 Fold-Change and the Y-axis is log10p-value. Red (up-regulated) and blue (down-regulated) points indicated the differential expression IncRNA. (B) Volcano plots of differentially expressed mRNAs. (C) Differentially expressed IncRNAs hierarchically clustered, each column represents a sample and every row represents a IncRNA. Red indicates high relative expression and blue indicates low relative expression. (D) Differentially expressed mRNAs hierarchically clustered. 
(A)

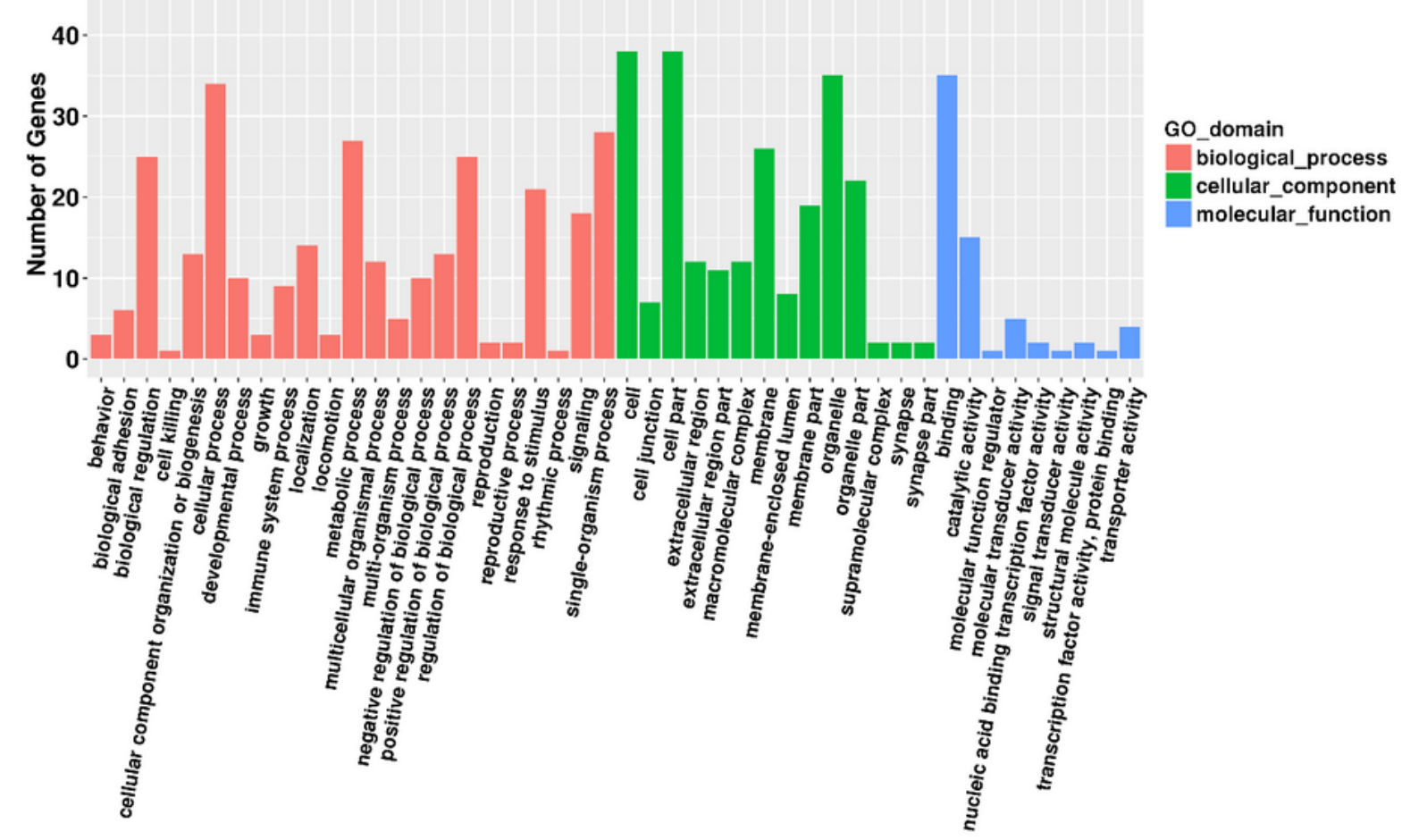

(B)

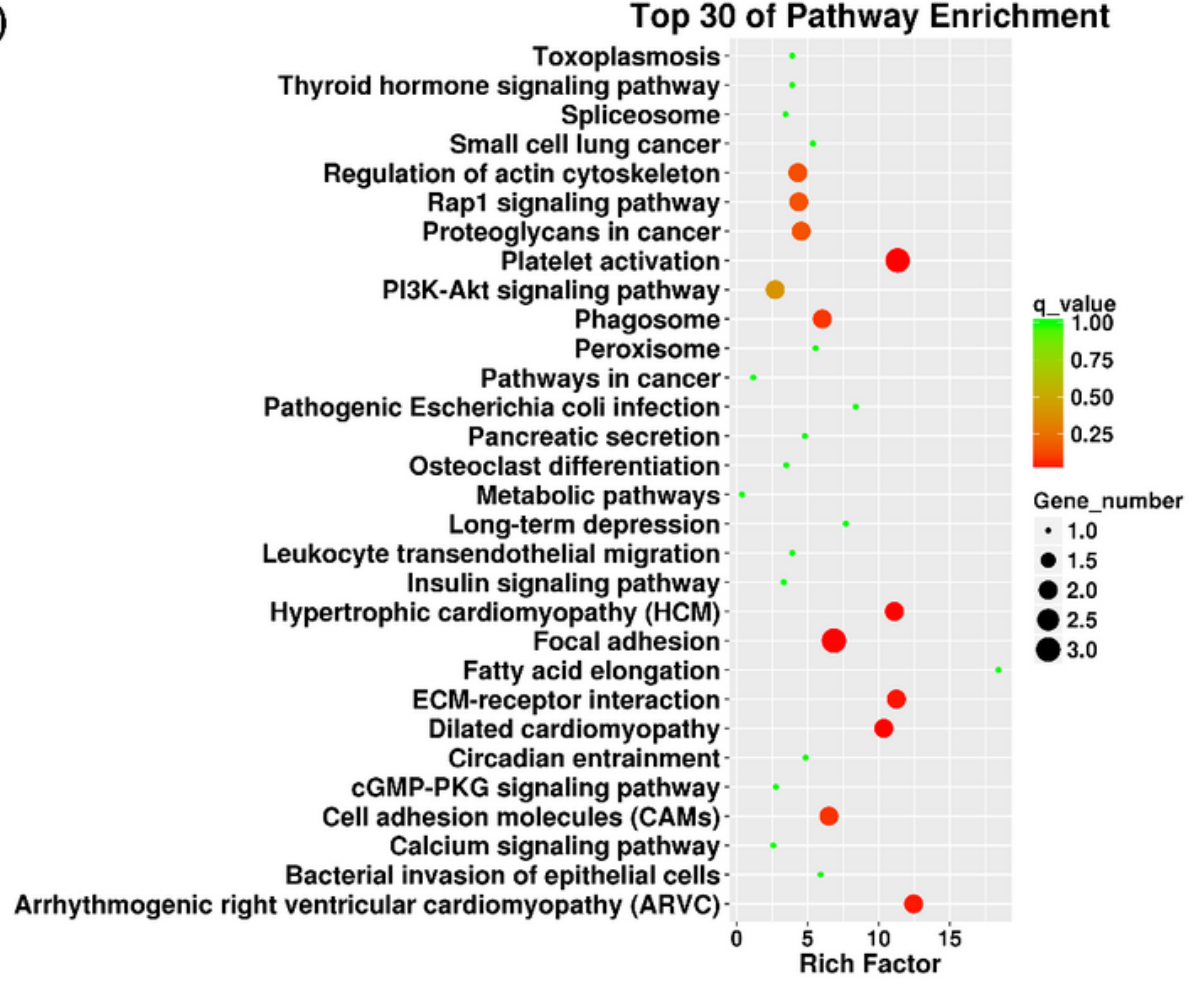

\section{Figure 2}

GO and KEGG pathway enrichment analysis. (A) GO enrichment contains three terms: cellular component, molecular function, and biological process. (B) the top 30 enriched KEGG pathways. 


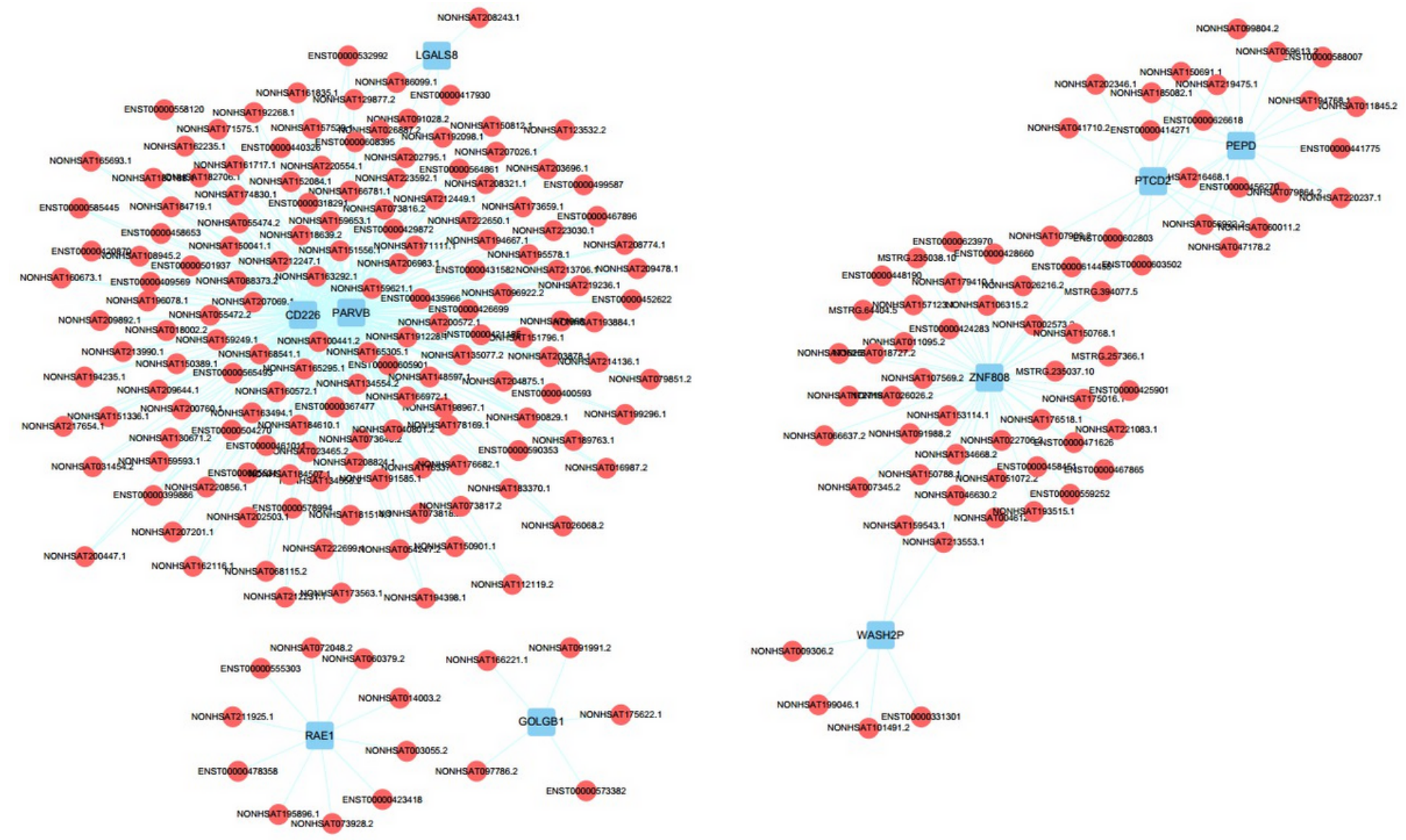

\section{Figure 3}

IncRNA-mRNA co-expression network. Red indicates IncRNAs and blue indicates mRNAs. the top 2 hub genes are CD226 and PARVB.
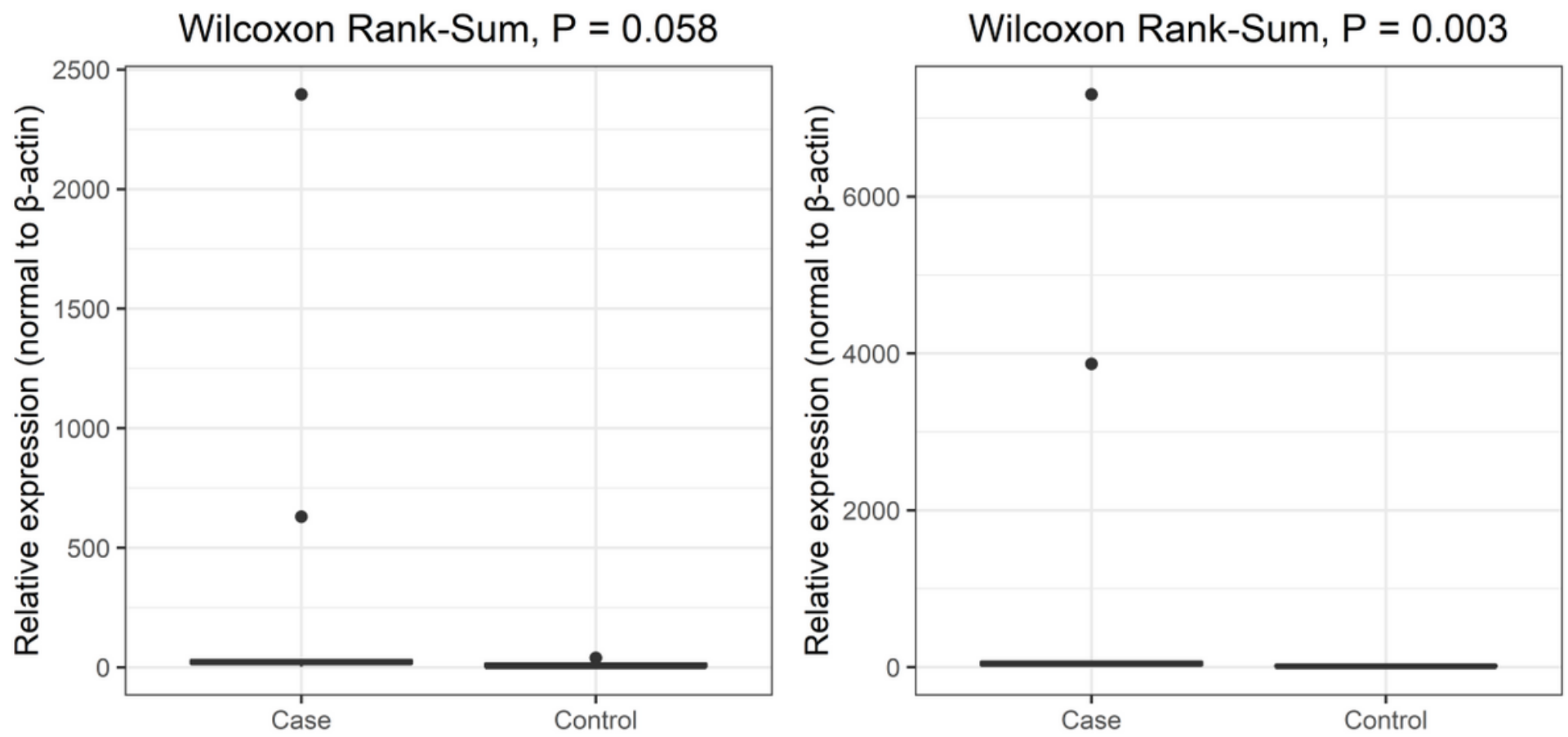
Figure 4

Relative levels of mRNAs and IncRNAs in 10 cases and 10 controls. Using qRT-PCR to calculate the relative fold-change to the house-keeping gene ( $\beta$-action). From left to right are IncRNA ENST00000590604 and CD226.

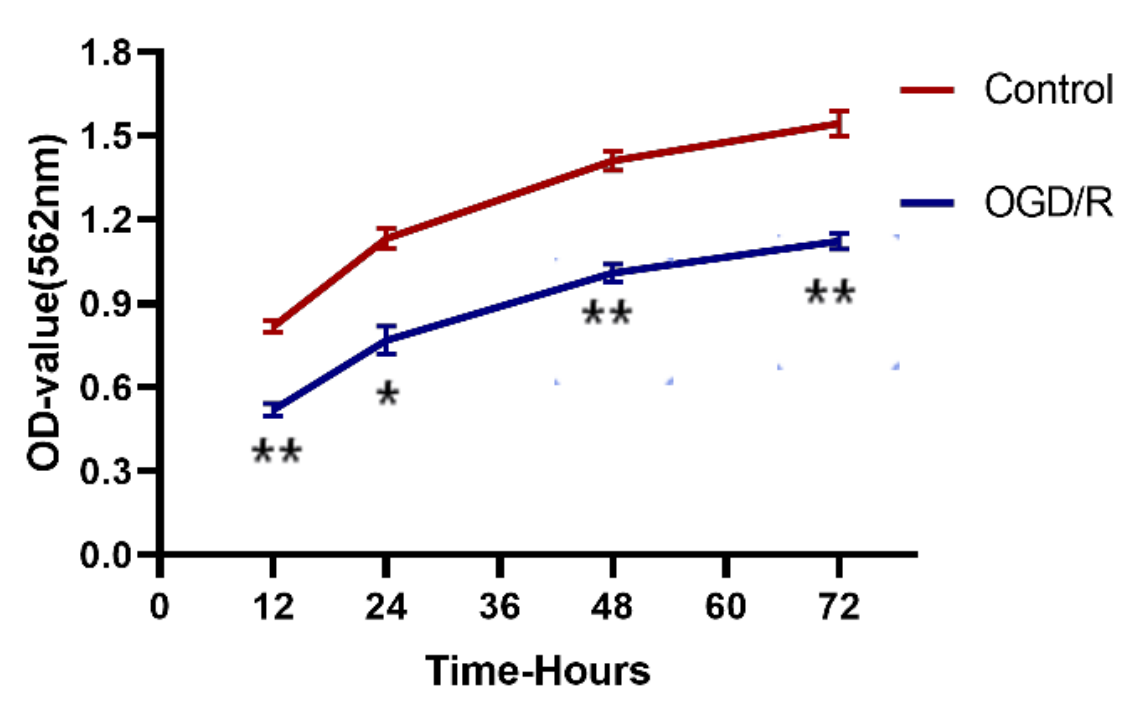

A

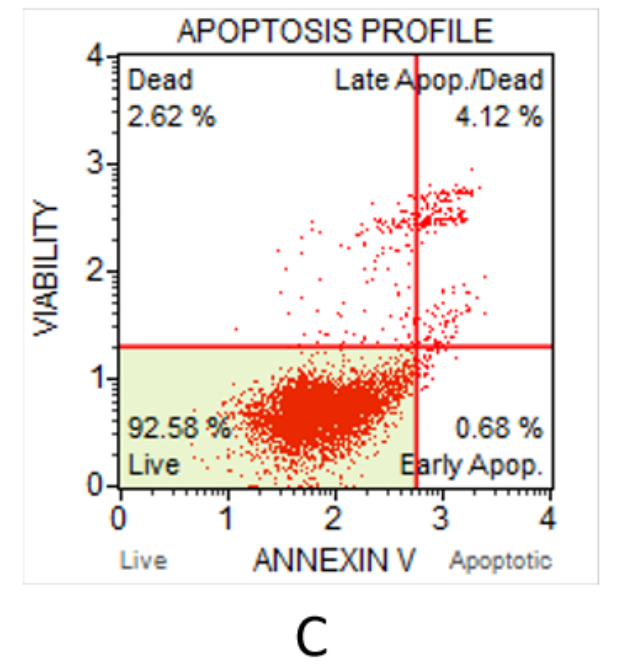

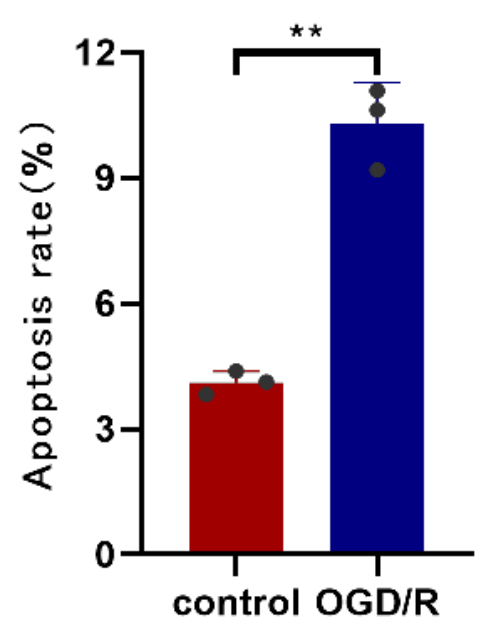

B

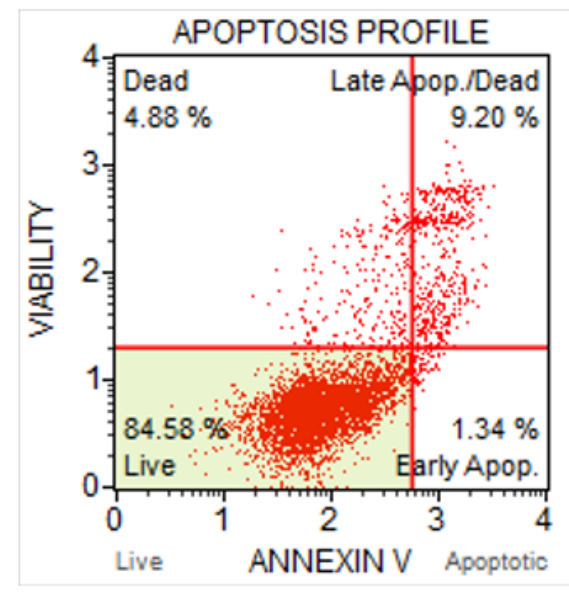

D

\section{Figure 5}

The effect of OGD/R on proliferation and apoptosis rate of HBMECs. (A) HBMECs' viability was declined after OGD/R compared with control group ( ${ }^{*} \mathrm{P}<0.05$, ${ }^{\star *} \mathrm{P}<0.01$ vs. Control group). (B) The apoptosis rate of $\mathrm{OGD} / \mathrm{R}$ group was increased compared with control group ( $* * \mathrm{P}<0.01$ vs. Control group). (C) Control group apoptosis results. (D) OGD/R group apoptosis results. 


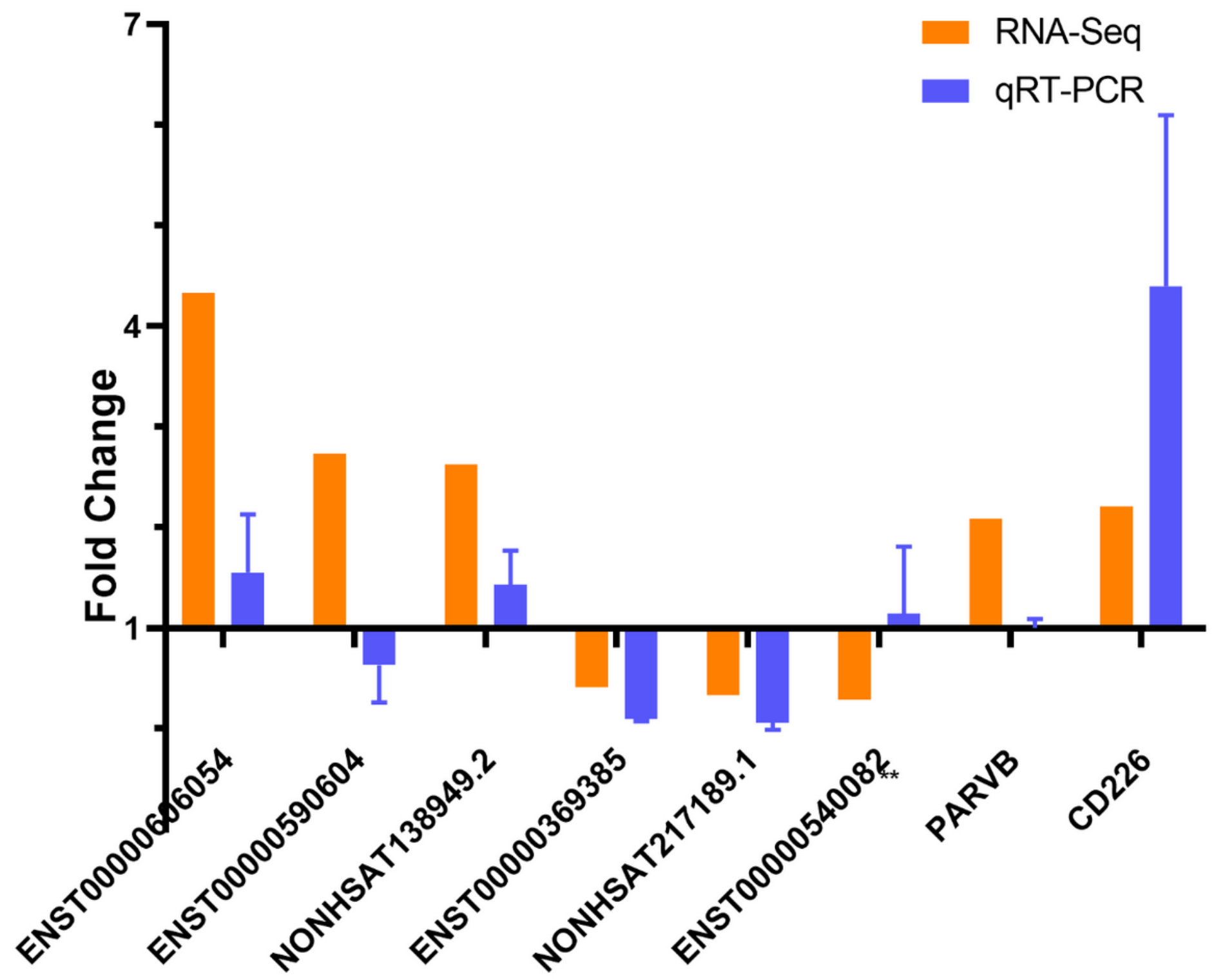

Figure 6

qRT-PCR validation $(n=3)$ of DE genes compared to RNA-Seq $(n=8)$. Yellow represents the expression of DE IncRNAs and DE mRNAs in the ISH complicated with stroke group veeq, blue represents the expression of DE IncRNAs and DE mRNAs in the Ang $\otimes$ and OGD/R group versusr the ISH group by RNA-S control group by qRT-PCR 